\title{
Analyse des déformées modales d'un treillis par la méthode des éléments finis
}

\section{Analysis of the modal deformations of a truss by the finite element method}

\author{
Trésor Kanyiki ${ }^{1}$, Jean Paul Katond ${ }^{2}$, François Ntambwe ${ }^{3}$ \\ ${ }^{1}$ Département d'électromécanique, Faculté Polytechnique, Université de Lubumbashi, République démocratique du \\ Congo, tresorkanyiki@gmail.com \\ ${ }^{2}$ Département d'électromécanique, Faculté Polytechnique, Université de Lubumbashi, République démocratique du \\ Congo, jpkatond@hotmail.com \\ ${ }^{3}$ Département d'électromécanique, Faculté Polytechnique, Université de Lubumbashi, République démocratique du \\ Congo, k_ntambwe@yahoo.fr
}

\begin{abstract}
RÉSUMÉ. L'objectif de cet article est d'effectuer l'analyse modale du treillis par la méthode des éléments finis. Le treillis étudié possède 13 degrés de liberté, ce qui a conduit à des matrices masses et de raideur fastidieuse. L'outil informatique Matlab a permis d'effectuer le calcul matriciel et de réaliser la représentation graphique des déplacements des nœuds du treillis pour chaque mode de vibration. Cette représentation graphique illustre la déformée modale du treillis.

ABSTRACT. The purpose of this paper is to perform modal analysis of truss by finite element method. The truss studied has 13 degrees of freedom, which led to mass matrix and stiffness tedious. The Matlab computer tool made it possible to carry out the matrix calculation and to realize the graphical representation of the displacement of the nodes of the truss for each mode of vibration. This representation illustrates the modal deformation of the truss.

MOTS-CLÉS. Treillis, Eléments finis, Analyse modale, Matlab.

KEYWORDS. Truss, Finite element, Modal analysis, Matlab.
\end{abstract}

\section{Introduction}

Le treillis est une structure très utilisé en génie civil, en construction mécanique, en génie mécanique, ... il est constitué d'éléments barres qui sont sollicités en traction compression [GEN 11]. Dans l'ingénierie moderne, les structures présentent différentes sortes de déformations avant la rupture. On parle de l'instabilité mécanique et de secousses dévastatrices en génie mécanique. En aéronautique, il arrive que les moteurs explosent en plein vol ou un hélicoptère qui se désintègrent au sol [BAT 90]. Mais il est crucial d'évoquer la technique nécessaire pour comprendre tous ces phénomènes. Cette technique repose à la fois sur des méthodes expérimentales et des modèles théoriques, elle s'appelle : analyse modale [IMB 84]. Dans ce travail, nous effectuons l'analyse modale théorique du treillis. L'analyse modale est liée à la nature intrinsèque de l'objet. Que la structure présente un amortissement ou non, le concepteur souhaite connaitre les modes de vibration des caractéristiques de la structure [DEH 02]. Pour une structure à plusieurs degrés de liberté cette démarche est fastidieuse ; cet article illustre la puissance de la méthode des éléments finis dans l'analyse modale du treillis. Cette démarche conduit aux calculs matriciels rébarbatifs, Matlab a permis d'effectuer le calcul matriciel et de réaliser la présentation des déformations modales du treillis. 


\section{Modélisation élément fini d'une barre}

La méthode des éléments finis est considérée comme une procédure particulière de la méthode de RayleighRitz. Elle consiste à diviser une structure ou un corps déformable en nombre fini d'éléments généralement de géométrie simple (hexaèdre ou tétraèdre en tridimensionnel, quadrangle ou triangle en bidimensionnel, segment de droite en monodimensionnel) et de fonction structurale bien identifiée [GER 97]. Les fonctions d'interpolation du champ des déplacements sont choisies de façon à présenter les propriétés suivantes [IMB 84]:

- L'interpolation est effectuée par des fonctions continues par morceaux. Sur chaque élément, le champ de déplacement sera représenté par une superposition des fonctions simples, mais qui représentent le comportement structural de l'élément dans la structure. Les fonctions sont du type polynomial.

- Les fonctions d'interpolation sont choisies de manière que les coordonnées généralisées de la méthode de Rayleigh-Ritz soient des valeurs du champ des déplacements locaux.

Un élément fini est dit cinématiquement admissible s'il respecte ces deux conditions.

\subsection{Barre en extension}

Considerons une barre en extension soumise à une charge uniformement répartie $\mathrm{p}(\mathrm{t})$. La Figure 1 montre une barre subdivisée en $\mathrm{N}$ éléments de longueur 1. Sur un élément, on interpole le champ des déplacements linéairement par la formule suivante[TOU 84] :

$$
u(x, t)=u_{1}(t) \phi_{1}(x)+u_{2}(t) \phi_{2}(x)
$$

Où

$u_{1}(t), u_{2}(t)$ : sont les dégrés de liberté connecteurs qui correspondent aux déplacement axiaux des deux extrémités des nœuds ;

$\phi_{1}(t), \phi_{2}(t)$ : sont les fonctions de forme de l'élément qui choisies de telle manière que : $u(0, t)=u_{1}(t)$ et $u(l, t)=u_{2}(t)$.

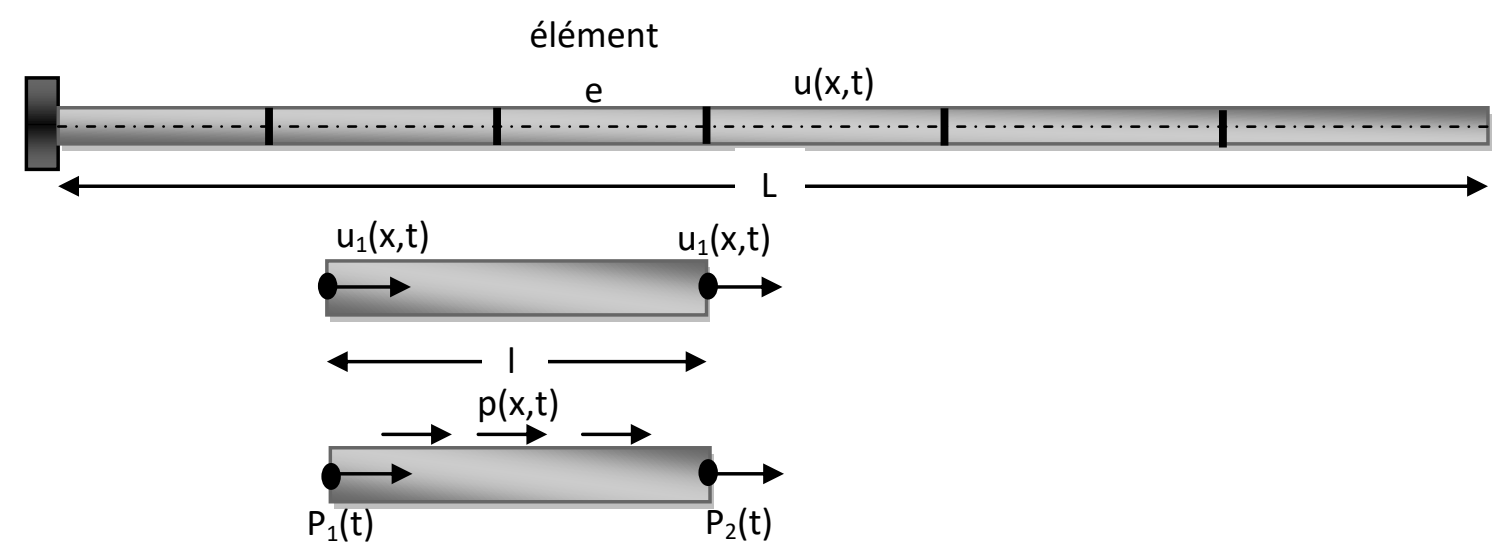

Figure 1. Barre en extension modélisée par N éléments finis.

La relation (1) prend la forme matricielle suivante :

$$
u(x, t)=N_{e}(x) q_{e}(t)
$$

Où

$N_{e}(t)=\left[\phi_{1}(x) \phi_{2}(x)\right]$ est une matrice des fonctions de forme de l'élément e ;

$q_{e}^{T}(t)=\left[\phi_{1}(x) \phi_{2}(x)\right]$ est une matrice des degrés de liberté de l'élément e.

Nous pouvons calculer ensuite successivement 
- sous formes quadratiques des matrices élémentaires de raideur et de masse, les énergies cinétiques et de déformation

$$
T_{e}=\frac{1}{2} \dot{q}_{e}^{T} M_{e} \dot{q}_{e} \quad \text { et } \quad v_{\mathrm{int}, e}=\frac{1}{2} \dot{q}_{e}^{T} K_{e} \dot{q}_{e}
$$

Avec

$$
\begin{aligned}
& M_{e}=\int_{0}^{l} m N_{e}^{T} N_{e} d x \\
& K_{e}=\int_{0}^{l} E A \frac{d N_{e}^{T}}{d x} \frac{d N_{e}}{d x} d x
\end{aligned}
$$

- le travail virtuel des forces extérieures :

$$
\delta v_{e x t, e}=-\delta q_{e}^{T} g_{e}(t)
$$

Avec les chargées généralisées $g_{e}(t)$ qui sont conjuguées aux déplacements $q_{e}(t)$.

$$
g_{e}(t)=\int_{0}^{l} N_{e}^{T} p(x, t) d x+\left[\begin{array}{l}
P_{1}(t) \\
P_{2}(t)
\end{array}\right]
$$

Dans la relation (7), le premier terme indique la discrétisation de la charge par unité de longueur et le second terme contient les charges d'extrémité de l'élément. Les charges d'extremité comportent deux contributions: les forces extérieures éventuelles et les forces de réaction avec les éléments voisins [GER 97]. On exprime la contribution de l'élément $e$ au principe de Hamilton, nous obtenons :

$$
\delta \int_{t_{1}}^{t_{2}}\left(\frac{1}{2} \dot{q}_{e}^{T} M_{e} \dot{q}_{e}-\frac{1}{2} q_{e}^{T} K_{e} q_{e}\right) d t+\delta q_{e}^{T} g_{e}=0
$$

\subsubsection{Processus d'assemblage}

Construisons la matrice des déplacements structuraux $q$ rassemblant les $(\mathrm{N}+1)$ déplacements nodaux (Figure 2), afin d'exprimer l'équilibre dynamique de l'ensemble du système de la Figure 1 [TOU 84].

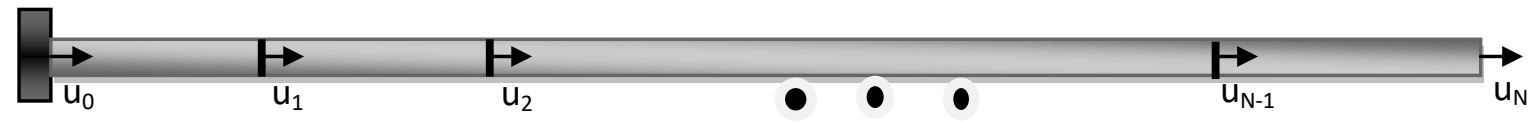

Figure 2. Déplacement nodaux du sytsème.

$$
\begin{gathered}
q^{T}=\left[\begin{array}{llllll}
q_{1} & q_{1} & q_{1} & q_{1} & \ldots & q_{N}
\end{array}\right] \\
q_{e}=L_{e} q
\end{gathered}
$$

Où $L_{e}$ est une matrice d'incidence.

L’équation variationnelle est construite par la sommation sur tous les éléments du système.

$$
\delta \int_{t_{1}}^{t_{2}} \sum_{e=1}^{N}\left[\frac{1}{2} \dot{q}_{e}^{T} M_{e} \dot{q}_{e}-\frac{1}{2} q_{e}^{T} K_{e} q_{e}\right] d t+\int_{t_{1}}^{t_{2}} \sum_{e=1}^{N} \delta q_{e}^{T} g_{e} d t=0
$$


En substituant l'expression (10) dans (10), nous obtenons :

$$
\delta \int_{t_{1}}^{t_{2}}\left\{\frac{1}{2} \dot{q}^{T}\left(\sum_{e=1}^{N} L_{e}^{T} M_{e} L_{e}\right) \dot{q}-\frac{1}{2} q^{T}\left(\sum_{e=1}^{N} L_{e}^{T} K_{e} L_{e}\right) q\right\} d t+\int_{t_{1}}^{t_{2}} \delta q^{T}\left(\sum_{e=1}^{N} L_{e}^{T} g_{e}\right) d t=0
$$

On définit alors :

- la matrice des masses structurale ou des masses du système assemblé

$$
M=\sum_{e=1}^{N} L_{e}^{T} M_{e} L_{e}
$$

- la matrice des raideurs structurale

$$
K=\sum_{e=1}^{N} L_{e}^{T} K_{e} L_{e}
$$

- le vecteur des charges structural

$$
g=\sum_{e=1}^{N} L_{e}^{T} g_{e}
$$

Par application les équations structurées sous la forme habituelle, nous obtenons les équations structurales discrétisées sous la forme habituelle :

$$
M \ddot{q}+K q=g(t)
$$

Pour éffectuer l'analyse modale, on considère que $g(t)=0$.

\section{Déformation modale du treillis}

Considérons une structure formée de barres telle que le treillis plan de la Figure 2, les membrures sont articulées aux niveau des nœuds et ne contribuent à la raideur global que par leurs extensions. A partir de l'élément de barre dejà présenté, les matrices de masse structurales et de raideur peuvent aisément être construites. Mais il faut exprimer les matrices élémentaires de chaque membrures dans un même système d'axes.
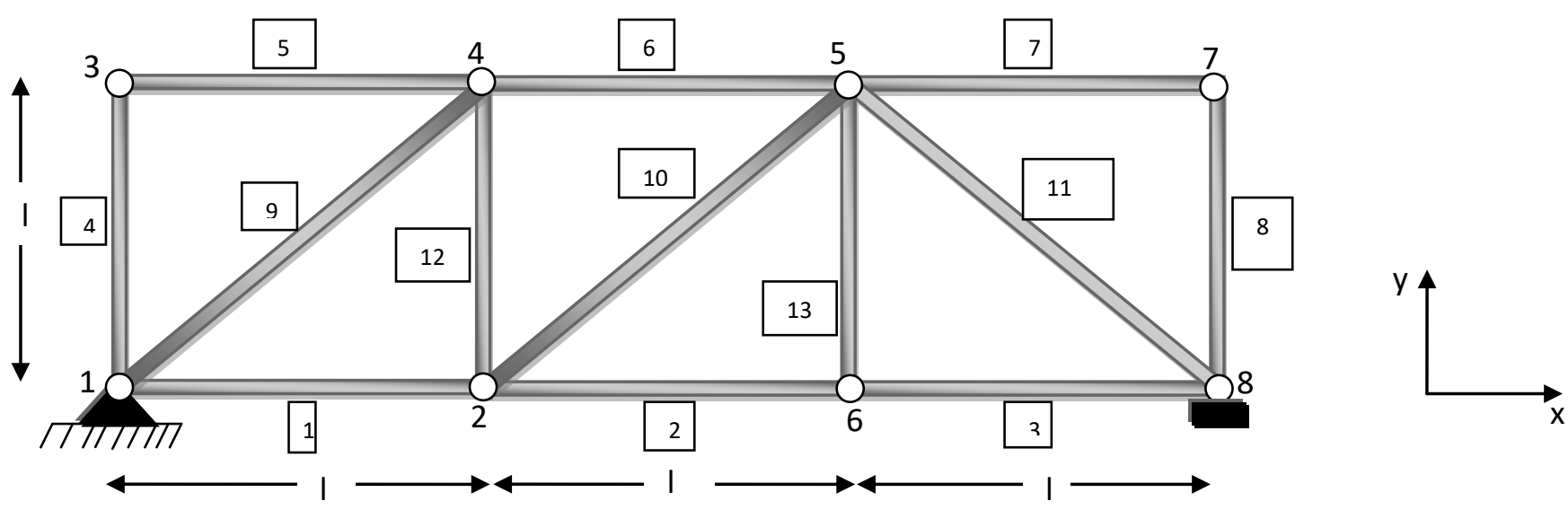

Figure 3. Structure en treillis. 


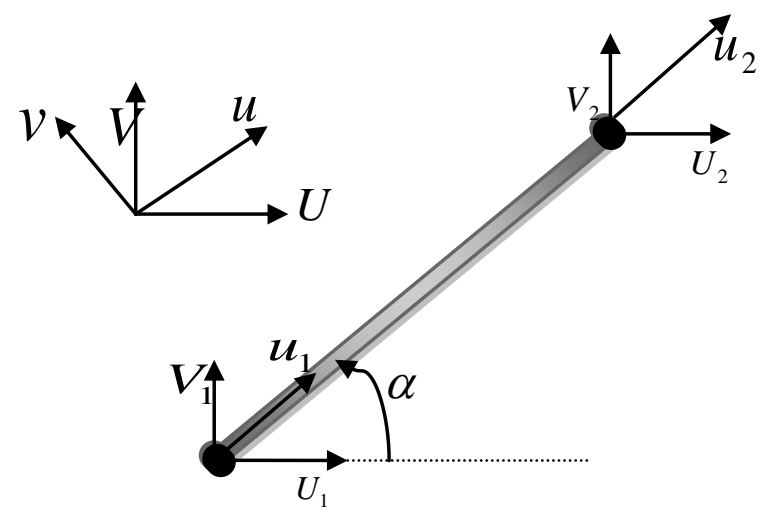

Figure 4. Elément de barre en axes quelconques.

A l'aide des données du treillis de la Figure 2, nous avons représenté le treillis sur Matlab.

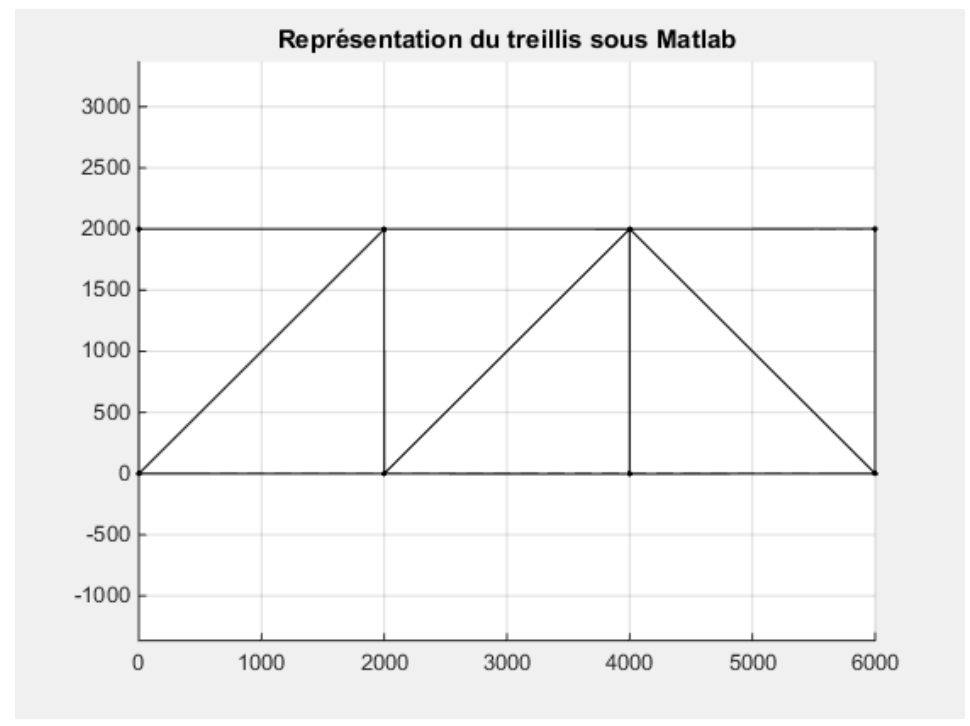

Figure 5. Treillis sur Matlab.

Considérons l'élément formant un angle $\alpha$ avec l'axe des x (Figure 3). La transformation suivante, exprime le passage des déplacements locaux en extension $u_{i}(i=1,2)$ aux déplacements structuraux $U_{i}, V_{i}$ s'exprime par la transformation :

$$
u_{i}=U_{i} \cos \alpha+V_{i} \sin \alpha \quad i=1,2
$$

Ce qui donne lieu à la transformation matricielle suivante :

$$
\left[\begin{array}{l}
u_{1} \\
u_{2}
\end{array}\right]=\left[\begin{array}{cccc}
\cos \alpha & \sin \alpha & 0 & 0 \\
0 & 0 & \cos \alpha & \sin \alpha
\end{array}\right]\left[\begin{array}{l}
U_{1} \\
V_{1} \\
U_{2} \\
V_{2}
\end{array}\right]
$$

Soit

$$
q_{e L}=R q_{e S}
$$


Avec $q_{e s}$ et $q_{e L}$ les déplacements respectivement de l'élément en axes structuraux et locaux, et $\mathrm{R}$ l'opération de rotation correspondante. Nous savons que l'énergie de déformation de l'élément est invariante de la rotation, nous avons :

$$
v_{\mathrm{int}, e}=\frac{1}{2} q_{e L}^{T} K_{e L} q_{e L}=\frac{1}{2} q_{e S}^{T} K_{e s} q_{e s}
$$

D'où la transformation de la matrice de raideur

$$
K_{e S}=R^{T} K_{e L} R
$$

En ce qui concerne l'énergie cinétique, son expression est complétée par le terme d'énergie cinétique transversale :

$$
\begin{aligned}
\tau_{e} & =\frac{1}{2} \int_{0}^{l} m\left(\dot{u}^{2}+\dot{v}^{2}\right) d x \\
& =\frac{1}{2} \int_{0}^{l} m\left(\dot{U}^{2}+\dot{V}^{2}\right) d x
\end{aligned}
$$

Et il en découle que la matrice de masse élémentaire est invariante vis-à-vis du changement d'axes. Par utilisation des mêmes fonctions de forme linéaires pour $v$ que pour $u$, nous obtenons pour l'élément de caractéristiques uniformes les matrices suivantes :

$$
K_{e S}=\frac{E A}{l}\left[\begin{array}{cccc}
\cos ^{2} \alpha & & \text { Sym. } & \\
\cos \alpha \sin \alpha & \sin ^{2} \alpha & & \\
-\cos \alpha & -\cos \alpha \sin \alpha & \cos ^{2} \alpha & \\
-\cos \alpha \sin \alpha & -\sin ^{2} \alpha & \cos \alpha \sin \alpha & \sin ^{2} \alpha
\end{array}\right]
$$

Et

$$
M_{e S}=\frac{m l}{6}\left[\begin{array}{cccc}
2 & & \text { Sym. } \\
0 & 2 & & \\
1 & 0 & 2 & \\
0 & 1 & 0 & 2
\end{array}\right]
$$

L'assemblage des matrices de masse structurale et de raideur s'organise de la façon suivante [DEH 02] :

a. Premièrement, on commence d'abord par identifier les degrés de liberté structuraux du système, ils sont collectés dans la matrice des déplacements structuraux en tenant compte des conditions aux limites.

$$
\begin{aligned}
& q^{T}=\left[\begin{array}{lllllllllllll}
U_{2} & V_{2} & U_{3} & V_{3} & U_{4} & V_{4} & U_{5} & V_{5} & U_{6} & V_{6} & U_{7} & V_{7} & U_{8}
\end{array}\right] \\
& =\left[\begin{array}{lllllllllllll}
q_{1} & q_{2} & q_{3} & q_{4} & q_{5} & q_{6} & q_{7} & q_{8} & q_{9} & q_{10} & q_{11} & q_{12} & q_{13}
\end{array}\right]
\end{aligned}
$$

b. Deuxièmement, on construit le vecteur de localisation pour chaque élément, ce vecteur établi une correspondance entre degrés de liberté structuraux et degrés de liberté élémentaires :

$$
\text { locel }(e, j)=\left\{\begin{array}{l}
\text { positiondu degré } q_{e, j} \text { dans } \\
\text { le vecteur structural } q
\end{array}\right.
$$

Dans cet article, nous avons écrit un programme sur Matlab qui permet d'assembler les matrices de masse et de raideur.

En utilisant la Figure 5 et la relation (26), nous obtenons le Tableau 1. 


\begin{tabular}{|c|c|}
\hline Elément & Vecteur \\
\hline 1 & $\left(\begin{array}{llll}0 & 0 & 1 & 2\end{array}\right)$ \\
\hline 2 & $\left(\begin{array}{llll}1 & 2 & 3 & 4\end{array}\right)$ \\
\hline 3 & $\left(\begin{array}{llll}3 & 4 & 5 & 0\end{array}\right)$ \\
\hline 4 & $\left(\begin{array}{llll}0 & 0 & 6 & 7\end{array}\right)$ \\
\hline 5 & $\left(\begin{array}{llll}0 & 0 & 8 & 9\end{array}\right)$ \\
\hline 6 & $\left(\begin{array}{llll}6 & 7 & 8 & 9\end{array}\right)$ \\
\hline 7 & $\left(\begin{array}{llll}1 & 2 & 8 & 9\end{array}\right)$ \\
\hline 8 & $\left(\begin{array}{llll}8 & 9 & 10 & 11\end{array}\right)$ \\
\hline 9 & $\left(\begin{array}{llll}1 & 2 & 10 & 11\end{array}\right)$ \\
\hline 10 & $\left(\begin{array}{llll}5 & 0 & 10 & 11\end{array}\right)$ \\
\hline 11 & $\left(\begin{array}{llll}10 & 11 & 12 & 13\end{array}\right)$ \\
\hline 12 & $\left(\begin{array}{llll}5 & 0 & 10 & 11\end{array}\right)$ \\
\hline 13 & $\left(\begin{array}{llll}5 & 0 & 12 & 13\end{array}\right)$ \\
\hline
\end{tabular}

Tableau 1. Vecteur de localisation des barres du treillis

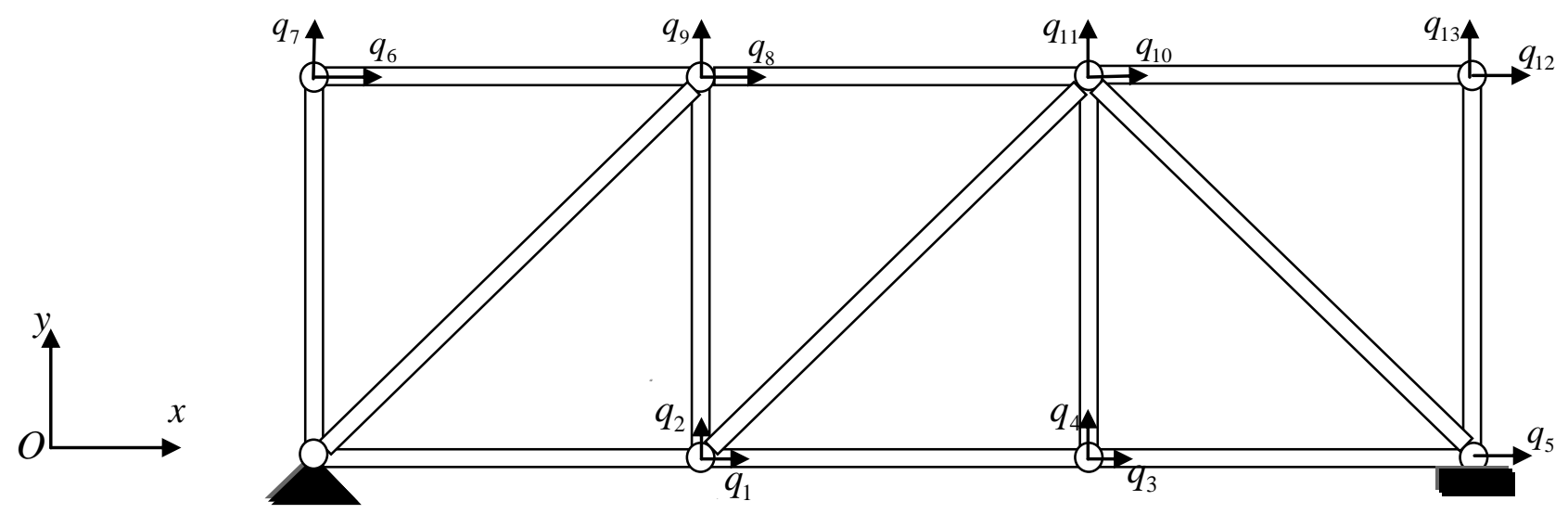

Figure 6. Treillis.

En respectant la numérotation des degrés de liberté pour chaque élément, nous avons la Figure 6.

Avec: $A=500 \mathrm{~mm}^{2} ; E=7000 \mathrm{~N} / \mathrm{mm}^{2} ; \mathrm{L}=200 \mathrm{~mm}$ pour les éléments $1,2,3,4,6,7,8,10,11$ et 13, $\mathrm{L}=200 \sqrt{2} \mathrm{~mm}$ pour les éléments 5,9 et $12 . \mathrm{m}=2.7 \mathrm{~kg} / \mathrm{mm}$.

Par application des relations (23) et (24) on construit les matrices de raideur et de masse élémentaires ; compte tenu des répétitions et symétrie du treillis, nous avons :

- pour les éléments 1,2,3,5,6 et 7

$$
k=\frac{E A}{l}\left[\begin{array}{cccc}
1 & 0 & -1 & 0 \\
0 & 0 & 0 & 0 \\
-1 & 0 & 1 & 0 \\
0 & 0 & 0 & 0
\end{array}\right] \quad M=\frac{m l}{6}\left[\begin{array}{cccc}
2 & 0 & 1 & 0 \\
0 & 2 & 0 & 1 \\
1 & 0 & 2 & 0 \\
0 & 1 & 0 & 2
\end{array}\right]
$$

- pour les éléments 4,8,12 et 13 
$k=\frac{E A}{l}\left[\begin{array}{cccc}0 & 0 & 0 & 0 \\ 0 & 1 & 0 & -1 \\ 0 & 0 & 0 & 0 \\ 0 & -1 & 0 & 0\end{array}\right]$

$M=\frac{m l}{6}\left[\begin{array}{cccc}2 & 0 & 1 & 0 \\ 0 & 2 & 0 & 1 \\ 1 & 0 & 2 & 0 \\ 0 & 1 & 0 & 2\end{array}\right]$

- pour les éléments 9 et 10

$k=\frac{E A}{2 l \sqrt{2}}\left[\begin{array}{cccc}1 & 1 & -1 & -1 \\ 1 & 1 & -1 & -1 \\ -1 & -1 & 1 & 1 \\ -1 & -1 & 1 & 1\end{array}\right]$

$M=\frac{m l \sqrt{2}}{6}\left[\begin{array}{llll}2 & 0 & 1 & 0 \\ 0 & 2 & 0 & 1 \\ 1 & 0 & 2 & 0 \\ 0 & 1 & 0 & 2\end{array}\right]$

- pour l'élément 11

$$
k=\frac{E A}{2 l \sqrt{2}}\left[\begin{array}{cccc}
1 & -1 & -1 & 1 \\
-1 & 1 & 1 & -1 \\
-1 & 1 & 1 & -1 \\
1 & -1 & -1 & 1
\end{array}\right]
$$$$
M=\frac{m l \sqrt{2}}{6}\left[\begin{array}{llll}
2 & 0 & 1 & 0 \\
0 & 2 & 0 & 1 \\
1 & 0 & 2 & 0 \\
0 & 1 & 0 & 2
\end{array}\right]
$$

A l'aide du tableau 1 et de la matrice de raideur de chaque élément, nous avons écrit un programme sur Matlab qui a permi d'assembler les matrices de raideur. Cette matrice de raideur nous l'avons multipliée au vecteur des paramètres de configuration et nous obtenons le système suivant :

$$
\left(\begin{array}{cccccccccccccccc}
1.3636 & 0.3536 & -1 & 0 & 0 & 0 & 0 & 0 & 0 & 0 & -0.3536 & -0.3536 & 0 & 0 & 0 & 0 \\
0.3536 & 1.3635 & 0 & 0 & 0 & 0 & 0 & 0 & 0 & -1 & -0.3536 & -0.3536 & 0 & 0 & 0 & 0 \\
-1 & 0 & 2.3536 & 0.3536 & -1 & 0 & 0 & 0 & 0 & 0 & 0 & 0 & -0.3536 & -0.3536 & 0 & 0 \\
0 & 0 & 0.3536 & 1.3536 & 0 & 0 & 0 & 0 & 0 & 0 & 0 & -1 & -0.3536 & -0.3536 & 0 & 0 \\
0 & 0 & -1 & 0 & 2 & 0 & -1 & 0 & 0 & 0 & 0 & 0 & 0 & 0 & 0 & 0 \\
0 & 0 & 0 & 0 & 0 & 1 & 0 & 0 & 0 & 0 & 0 & 0 & 0 & -1 & 0 & 0 \\
0 & 0 & 0 & 0 & -1 & 0 & 1.3536 & -0.3536 & 0 & 0 & 0 & 0 & -0.3536 & 0.3536 & 0 & 0 \\
0 & 0 & 0 & 0 & 0 & 0 & -0.3536 & 1.3536 & 0 & 0 & 0 & 0 & 0.3536 & -0.3536 & 0 & -1 \\
0 \\
0 & 0 & 0 & 0 & 0 & 0 & 0 & 0 & 1 & 0 & -1 & 0 & 0 & 0 & 0 & 0 \\
q_{1} \\
q_{2} \\
q_{3} \\
q_{4} \\
q_{5} \\
0 \\
-0.3536 & -1 & 0 & 0 & 0 & 0 & 0 & 0 & 0 & 1 & 0 & 0 & 0 & 0 & 0 & 0 \\
-0.3536 & -0.3536 & 0 & 0 & 0 & 0 & 0 & 0 & -1 & 0 & 2.3536 & 0.3536 & -1 & 0 & 0 & 0 \\
0 & 0 & -0.3536 & -0.3536 & 0 & 0 & -0.3536 & 0.3536 & 0 & 0 & -1 & 0 & 2.701 & 0 & -1 & 0 \\
0 & 0 & -0.3536 & -0.3536 & 0 & -1 & 0.3536 & -0.3536 & 0 & 0 & 0 & 0 & 0 & 1.7071 & 0 & 0 \\
0 & 0 & 0 & 0 & 0 & 0 & 0 & 0 & 0 & 0 & 0 & 0 & -1 & 0 & 1 & 0 \\
0 & 0 & 0 & 0 & 0 & 0 & 0 & -1 & 0 & 0 & 0 & 0 & 0 & 0 & 0 & 1 \\
q_{7} \\
q_{8} \\
q_{9} \\
q_{10} \\
q_{11} \\
q_{12} \\
q_{13}
\end{array}\right)
$$

En éliminant les lignes et les colonnes qui correspondent au degré de liberté 0 dans le vecteur identifiant les degrés de liberté structuraux (ou vecteur des paramètres de configuration) ; nous obtenons la matrice de raideur $\mathrm{K}$ du treillis. 


$$
K=\frac{E A}{l}\left(\begin{array}{ccccccccccccc}
2.3536 & 0.3536 & -1 & 0 & 0 & 0 & 0 & 0 & 0 & -0.3536 & -0.3536 & 0 & 0 \\
0.3536 & 1.3536 & 0 & 0 & 0 & 0 & 0 & 0 & -1 & -0.3536 & -0.3536 & 0 & 0 \\
-1 & 0 & 2 & 0 & -1 & 0 & 0 & 0 & 0 & 0 & 0 & 0 & 0 \\
0 & 0 & 0 & 1 & 0 & 0 & 0 & 0 & 0 & 0 & -1 & 0 & 0 \\
0 & 0 & -1 & 0 & 1.3536 & 0 & 0 & 0 & 0 & -0.3536 & 0.3536 & 0 & 0 \\
0 & 0 & 0 & 0 & 0 & 1 & 0 & -1 & 0 & 0 & 0 & 0 & 0 \\
0 & 0 & 0 & 0 & 0 & 0 & 1 & 0 & 0 & 0 & 0 & 0 & 0 \\
0 & 0 & 0 & 0 & 0 & -1 & 0 & 2.3536 & 0.3536 & -1 & 0 & 0 & 0 \\
0 & -1 & 0 & 0 & 0 & 0 & 0 & 0.3536 & 1.3536 & 0 & 0 & 0 & 0 \\
-0.3536 & -0.3536 & 0 & 0 & -0.3536 & 0 & 0 & -1 & 0 & 2.701 & 0 & -1 & 0 \\
-0.3536 & -0.3536 & 0 & -1 & 0.3536 & 0 & 0 & 0 & 0 & 0 & 1.701 & 0 & 0 \\
0 & 0 & 0 & 0 & 0 & 0 & 0 & 0 & 0 & -1 & 0 & 1 & 0 \\
0 & 0 & 0 & 0 & 0 & 0 & 0 & 0 & 0 & 0 & 0 & 0 & 1
\end{array}\right)
$$

L'assemblage de la matrice des masses se fait de la même façon que celle de la matrice de raideur $\left(\begin{array}{cccccccccccccccc}6.8284 & 0 & 1 & 0 & 0 & 0 & 0 & 0 & 1 & 0 & 1.4142 & 0 & 0 & 0 & 0 & 0 \\ 0 & 6.8284 & 0 & 1 & 0 & 0 & 0 & 0 & 0 & 1 & 0 & 1.4142 & 0 & 0 & 0 & 0 \\ 100 & 0 & 8.8284 & 0 & 1 & 0 & 0 & 0 & 0 & 0 & 1 & 0 & 1.4142 & 0 & 0 & 0 \\ 0 & 1 & 0 & 8.8284 & 0 & 1 & 0 & 0 & 0 & 0 & 0 & 1 & 0 & 1.4142 & 0 & 0 \\ 0 & 0 & 1 & 0 & 6 & 0 & 1 & 0 & 0 & 0 & 0 & 0 & 1 & 0 & 0 & 0 \\ 0 & 0 & 0 & 1 & 0 & 6 & 0 & 1 & 0 & 0 & 0 & 0 & 0 & 1 & 0 & 0 \\ 0 & 0 & 0 & 0 & 1 & 0 & 6.8284 & 0 & 0 & 0 & 0 & 0 & 1.4142 & 0 & 1 & 0 \\ 0 & 0 & 0 & 0 & 0 & 1 & 0 & 6.8284 & 0 & 0 & 0 & 0 & 0 & 1.4142 & 0 & 1 \\ 1 & 0 & 0 & 0 & 0 & 0 & 0 & 0 & 4 & 0 & 1 & 0 & 0 & 0 & 0 & 0 \\ 0 & 1 & 0 & 0 & 0 & 0 & 0 & 0 & 0 & 4 & 0 & 1 & 0 & 0 & 0 & 0 \\ q_{1} \\ q_{2} \\ q_{3} \\ q_{4} \\ q_{5} \\ 0 \\ 0 & 1.4142 & 0 & 1 & 0 & 0 & 0 & 0 & 0 & 1 & 0 & 8.8284 & 0 & 1 & 0 & 0 \\ 0 & 0 & 1.4142 & 0 & 1 & 0 & 1.4142 & 0 & 0 & 0 & 1 & 0 & 11.6568 & 0 & 1 & 0 \\ 0 & 0 & 0 & 1.4142 & 0 & 1 & 0 & 1.4142 & 0 & 0 & 0 & 1 & 0 & 11.6568 & 0 & 1 \\ 0 & 0 & 0 & 0 & 0 & 0 & 1 & 0 & 0 & 0 & 0 & 0 & 1 & 0 & 4 & 0 \\ 0 & 0 & 0 & 0 & 0 & 0 & 0 & 1 & 0 & 0 & 0 & 0 & 0 & 1 & 0 & 4\end{array}\right)\left(\begin{array}{c}q_{7} \\ q_{7} \\ q_{8} \\ q_{9} \\ q_{10} \\ q_{11} \\ q_{12} \\ q_{13}\end{array}\right)$

Nous supprimons les lignes et les colonnes qui correspondent à zéro dans le vecteur de degré de liberté, nous obtenons la matrice masse :

$$
M=\frac{m l}{6}\left(\begin{array}{ccccccccccccc}
8.8284 & 0 & 1 & 0 & 0 & 0 & 0 & 1 & 0 & 1.4142 & 0 & 0 & 0 \\
0 & 8.8284 & 0 & 1 & 0 & 0 & 0 & 0 & 1 & 0 & 1.4142 & 0 & 0 \\
1 & 0 & 6 & 0 & 1 & 0 & 0 & 0 & 0 & 1 & 0 & 0 & 0 \\
0 & 1 & 0 & 6 & 0 & 0 & 0 & 0 & 0 & 0 & 1 & 0 & 0 \\
0 & 0 & 1 & 0 & 6.8284 & 0 & 0 & 0 & 0 & 1.4142 & 0 & 1 & 0 \\
0 & 0 & 0 & 0 & 0 & 4 & 0 & 1 & 0 & 0 & 0 & 0 & 0 \\
0 & 0 & 0 & 0 & 0 & 0 & 4 & 0 & 1 & 0 & 0 & 0 & 0 \\
1 & 0 & 0 & 0 & 0 & 1 & 0 & 8.8284 & 0 & 1 & 0 & 0 & 0 \\
0 & 1 & 0 & 0 & 0 & 0 & 1 & 0 & 8.8284 & 0 & 1 & 0 & 0 \\
1.4142 & 0 & 1 & 0 & 1.4142 & 0 & 0 & 1 & 0 & 11.6568 & 0 & 1 & 0 \\
0 & 1.4142 & 0 & 1 & 0 & 0 & 0 & 0 & 1 & 0 & 11.6568 & 0 & 1 \\
0 & 0 & 0 & 0 & 1 & 0 & 0 & 0 & 0 & 1 & 0 & 4 & 0 \\
0 & 0 & 0 & 0 & 0 & 0 & 0 & 0 & 0 & 0 & 1 & 0 & 4
\end{array}\right)
$$


Pour déterminer les caractéristiques modales du treillis, nous désignons le vecteur des paramètres de configuration par $\{\vec{q}\}$. L'équation du mouvement libre est :

$$
[M]\{\overrightarrow{\ddot{q}}\}+[K]\{\vec{q}\}=\{\overrightarrow{0}\}
$$

Nous considérons les solutions particulières dans de l'équation (33) dans lesquelles tous les paramètres de configuration vibrent en opposition de phase ou en phase. Ces solutions particulières se mettent sous la forme suivante :

$$
\begin{aligned}
& \{\vec{q}(t)\}=\{\vec{q}\} \cos (\omega t+\phi) \\
& \{\overrightarrow{\dot{q}}(t)\}=\{\vec{q}\}(-\omega) \sin (\omega t+\phi) \\
& \{\overrightarrow{\vec{q}}(t)\}=\{\vec{q}\}\left(-\omega^{2}\right) \cos (\omega t+\phi)
\end{aligned}
$$

Où $\{\vec{q}\}$ est un vecteur qui ne dépend pas explicitement du temps et qui décrit la forme de la vibration. En combinant les relations (34) et (35) ; et après simplification, nous obtenons l'expression algébrique suivante :

$$
\left([K]-\omega^{2}[M]\right)\{\vec{q}\}=\{\overrightarrow{0}\}
$$

Pour autant que le déterminant du système d'équation (35) soit nul, ce système admet des solutions non triviales.

$$
\operatorname{det}\left([K]-\omega^{2}[M]\right)=0
$$

Dans [GER 95] on explique que lorsque le nombre de degrés de liberté du système est compris entre 10 et 250, on peut utiliser les méthodes standards de résolution du problème aux valeurs propres.

En posant $\lambda=\omega^{2}$ et en calculant le déterminant de la relation (36), sachant que les matrices $[K]$ et $[M]$ sont données respectivement par les relations (31) et (32), nous obtenons l'équation caractéristique suivante :

$-9.5710 \times 10^{-4} \lambda^{13}+0.0622 \lambda^{12}-1.7679 \lambda^{11}+28.9898 \lambda^{10}-304.1219 \lambda^{9}+21391.1 \lambda^{8}-1027.2 \lambda^{7}+33624 \lambda^{6}-7356.8 \lambda^{5}$

$+103400 \lambda^{4}-87412 \lambda^{3}+40044 \lambda^{2}-8290 \lambda+557.0078=0$

A l'aide de la fonction « roots » de Matlab, nous avons trouvé les racines de l'équation (37);

$\lambda_{1}=12.53096937089212, \lambda_{2}=11.698004\left(29, \lambda_{3}=8.549756147, \lambda_{4}=6.771090074, \lambda_{5}=6.341820064\right.$,

$\lambda_{6}=4.834591126, \lambda_{7}=4.72027672, \lambda_{8}=4.2662072 Q, \lambda_{9}=2.71281519, \lambda_{10}=1.2586962 \mathrm{~b}$

$\lambda_{11}=0.854122378, \lambda_{12}=0.306371047$ et $\lambda_{13}=0.1243095 \mathrm{D}$.

A partir de chaque valeur propre, on déduit directement la valeur de la fréquence propre.

\section{Recherche des modes}

Par définition si $\{\vec{q}\}$ est un mode propre, nous avons :

$$
(-\lambda[M]+[K])\{\vec{q}\}=\{\hat{0}\}
$$

Pour le premier mode $\lambda_{1}=12.53096937029212$ du treillis, l'équation (38), devient : 


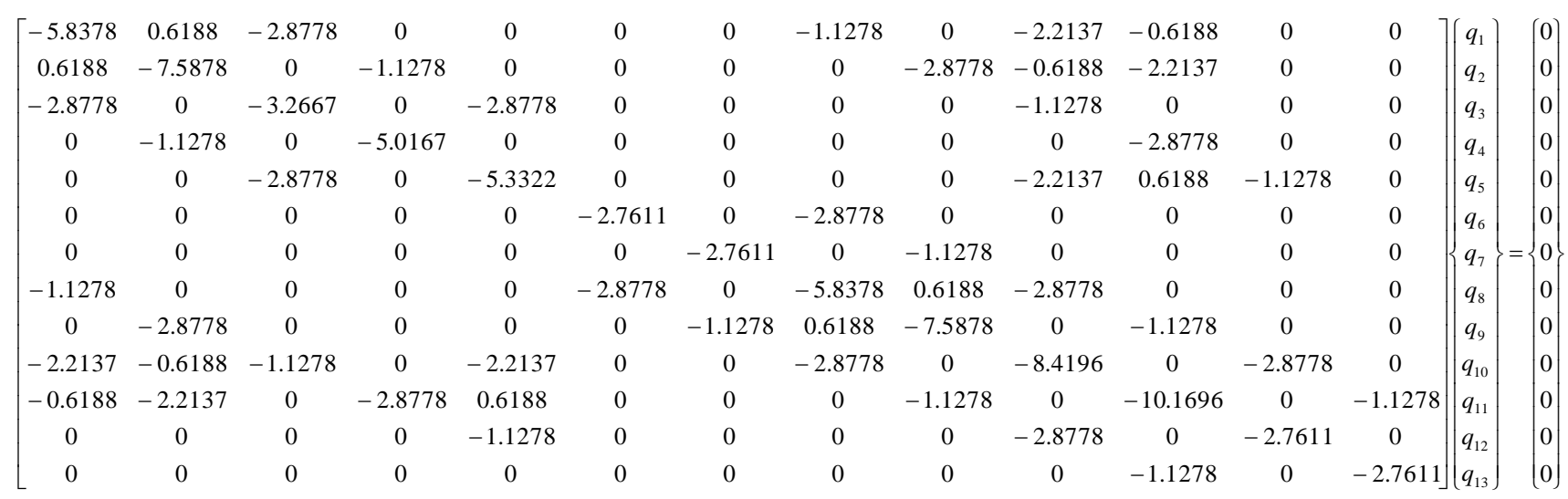

Ces 13 équations ne sont pas linéairement indépendantes car le déterminant du système d'équation est nul. Pour résoudre un tel système, une composante peut être choisie de manière arbitraire, par exemple la treizième égale à $0.0117\left(q_{13}=0.0117\right)$. A une constante multiplicative près, à partir de (39), nous obtenons le système suivant de 12 équations à 12 inconnues.

$$
\begin{aligned}
& -5.8378 q_{1}+0.6188 q_{2}-2.8778 q_{3}-1.1278 q_{8}-2.2137 q_{10}-0.6188 q_{11}=0 \\
& 0.6188 q_{1}-7.5878 q_{2}-1.1278 q_{4}-2.8778 q_{9}-0.6188 q_{10}-2.213 q_{11}=0 \\
& -2.8778 q_{1}-3.2667 q_{3}-2.8778 q_{5}-1.1278 q_{10}=0 \\
& -1.1278 q_{2}-5.0167 q_{4}-2.8778 q_{11}=0 \\
& -2.8778 q_{3}-5.3322 q_{5}-2.2137 q_{10}+0.6188 q_{11}-1.1278 q_{12}=0 \\
& -2.7611 q_{6}-2.8778 q_{8}=0 \\
& -2.7611 q_{7}-1.1278 q_{9}=0 \\
& -1.1278 q_{1}-2.8778 q_{6}-5.8378 q_{8}+0.6188 q_{9}-2.8778 q_{10}=0 \\
& -2.2778 q_{2}-1.1278 q_{7}+0.6188 q_{8}-7.5878 q_{9}-1.1278 q_{11}=0 \\
& -2.2137 q_{1}-0.6188 q_{2}-1.1278 q_{3}-2.2137 q_{5}-2.8778 q_{8}-8.4196 q_{10}-2.8778 q_{12}=0 \\
& -0.6188 q_{1}-2.2137 q_{2}-2.8778 q_{4}+0.6188 q_{5}-1.1278 q_{9}-10.1696 q_{11}=0.09 \\
& -1.1278 q_{5}-2.8778 q_{10}-2.7611 q_{12}=0
\end{aligned}
$$

Ce système d'équations est mis sous forme matricielle et résolu sur Matlab, les résultats sont : $q_{1}=4.52166 \mathrm{~mm}, q_{2}=0.5536 \mathrm{~mm}, q_{3}=-8.1919 \mathrm{~mm}, q_{4}=-0.1079 \mathrm{~mm}, q_{5}=4.9049 \mathrm{~mm}, q_{6}=1.6087 \mathrm{~mm}$, $q_{7}=0.1442 \mathrm{~mm}, q_{8}=-1.5435 \mathrm{~mm}, q_{9}=-0.3530 \mathrm{~mm}, q_{10}=-0.3255 \mathrm{~mm}, q_{11}=-0.028 \mathrm{~mm}, q_{12}=-1.6641 \mathrm{~mm}$ nous ajoutons $q_{13}=0.0117$.

La représentation des différents déplacements des nœuds sous Matlab, donne la déformée modale du treillis.

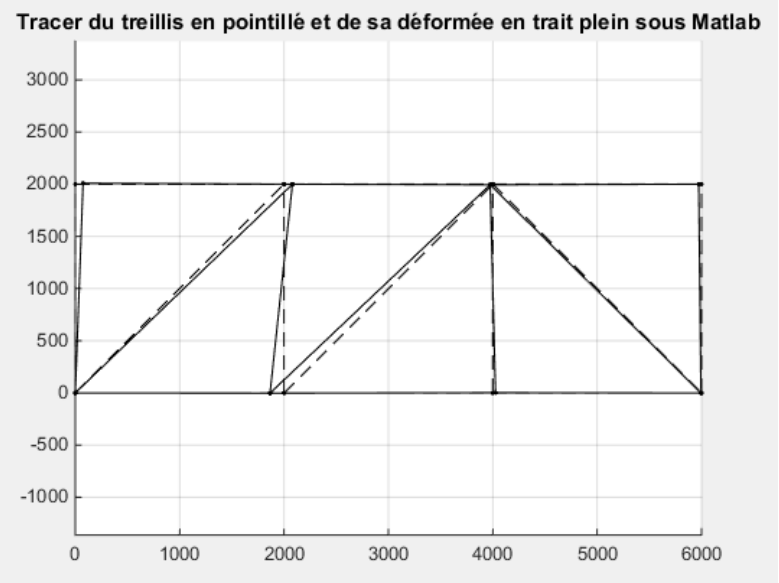

Figure 7. Mode 1.

De manière analogue, nous obtenons les déformées modales des modes propres du treillis. 


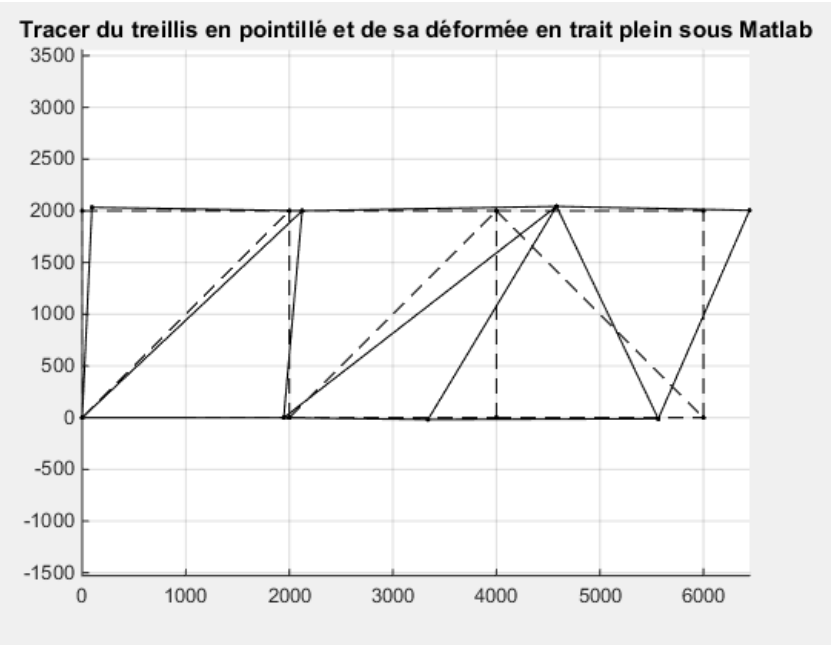

Figure 8. Mode 2.

$\lambda_{3}=8.549756147$

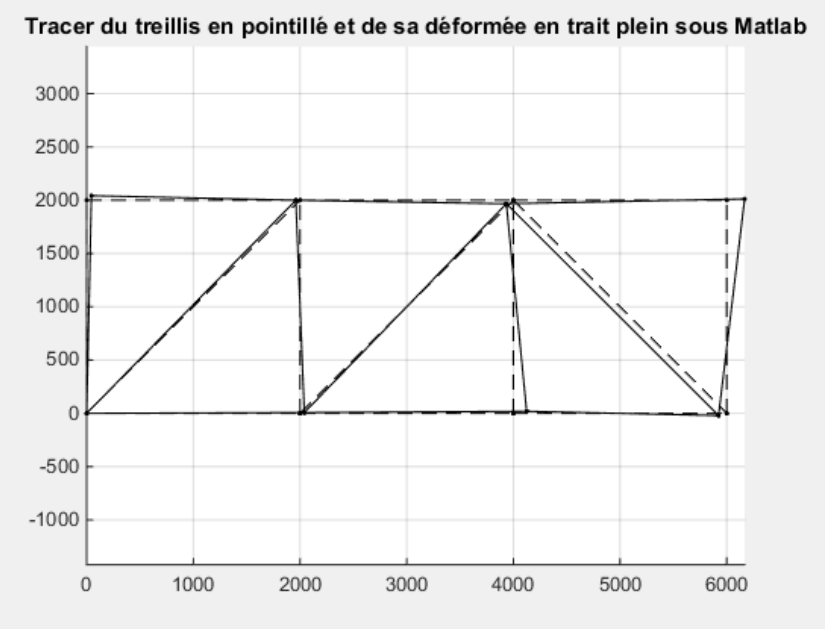

Figure 9. Mode 3.

$\lambda_{4}=6.771090074$

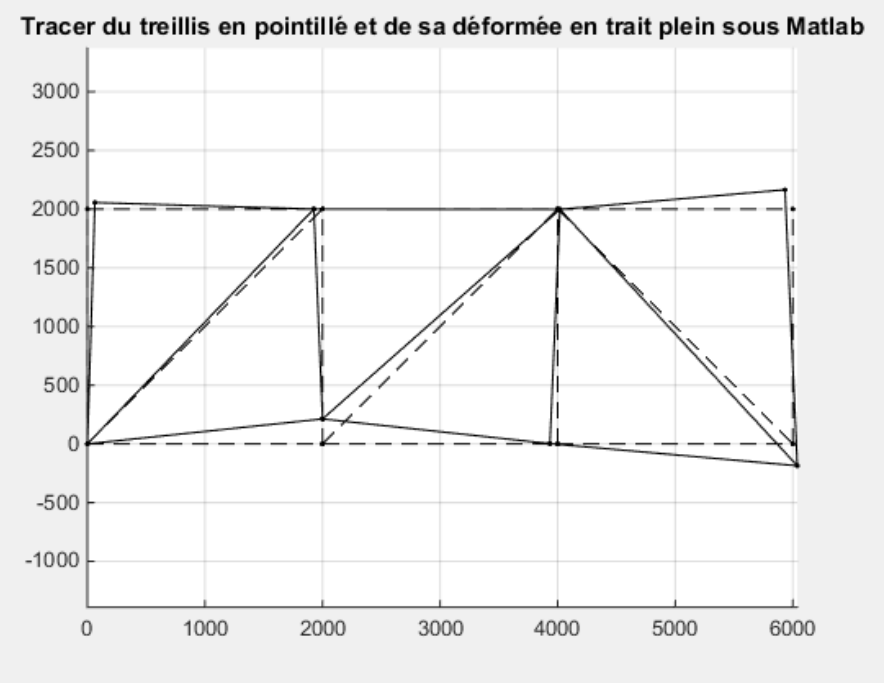

Figure 10. Mode 4.

$\lambda_{5}=6.341820064$ 


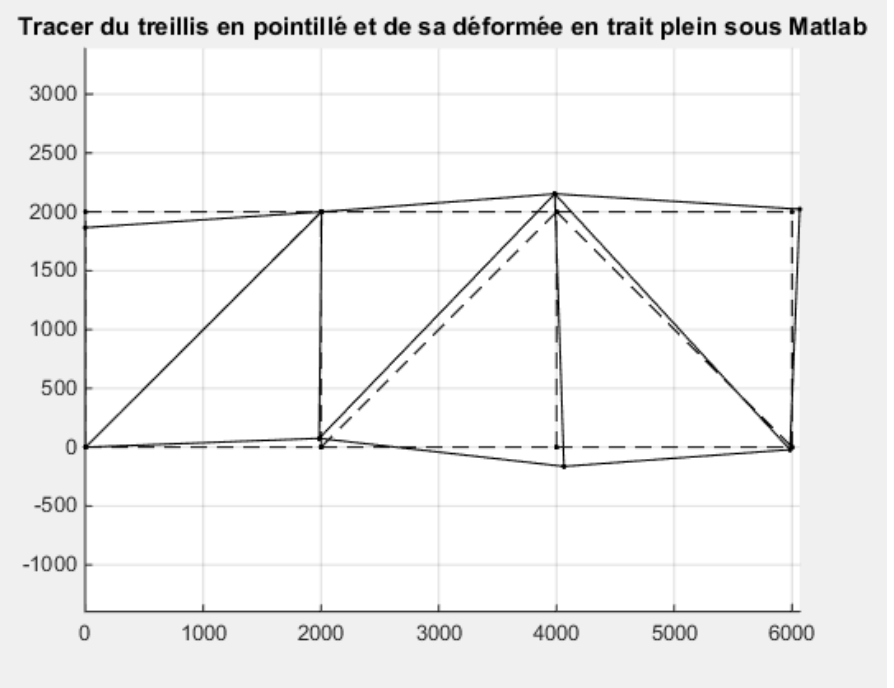

Figure 11. Mode 5.

$\lambda_{6}=4.834591126$

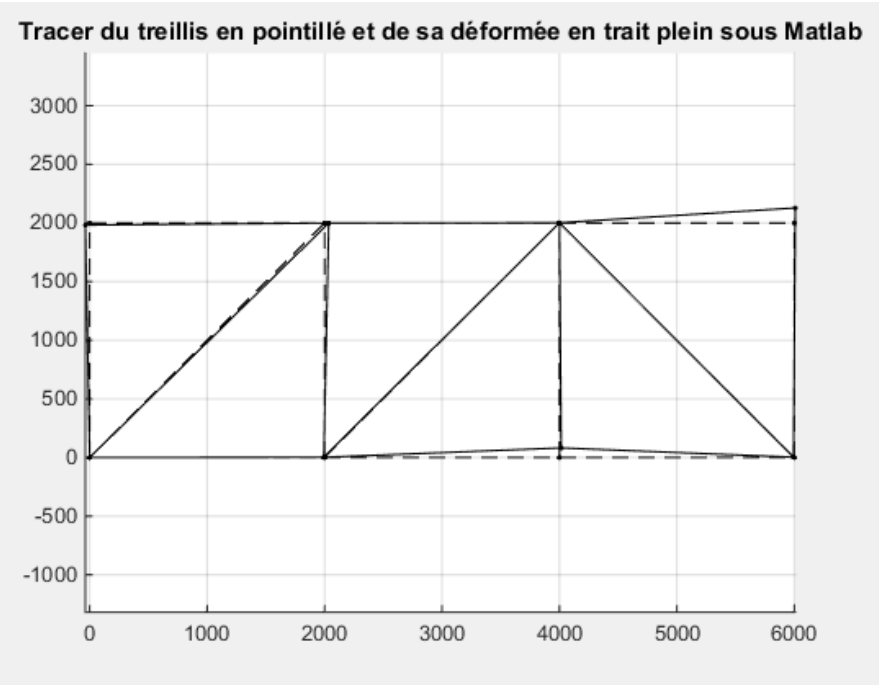

Figure 12. Mode 6.

$\lambda_{7}=4.72027672$

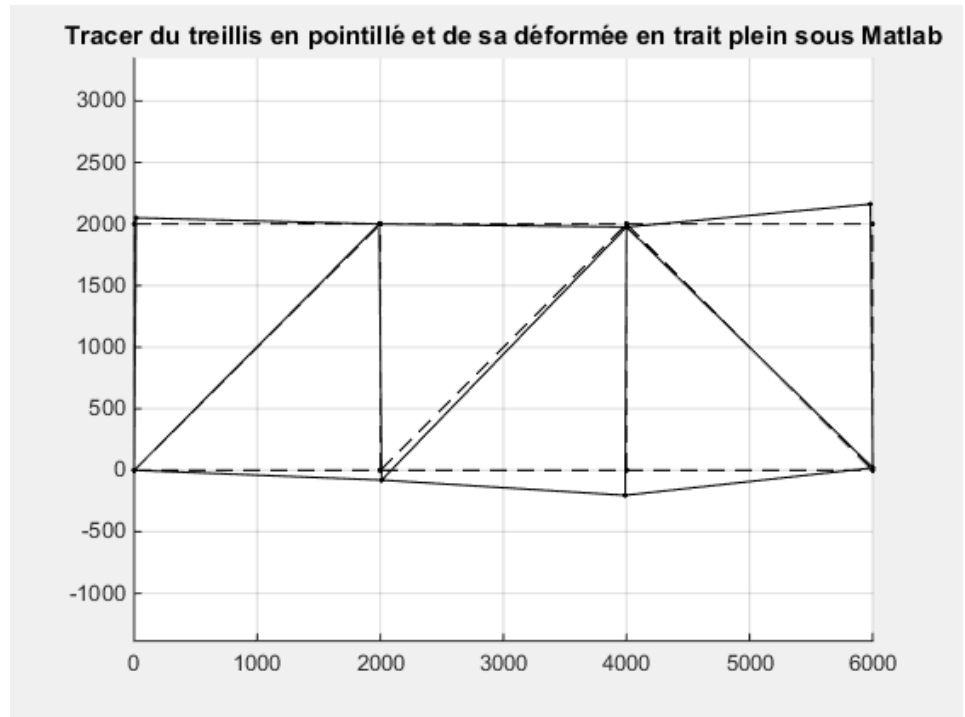

Figure 13. Mode 7.

$\lambda_{8}=4.2662072 Q$ 


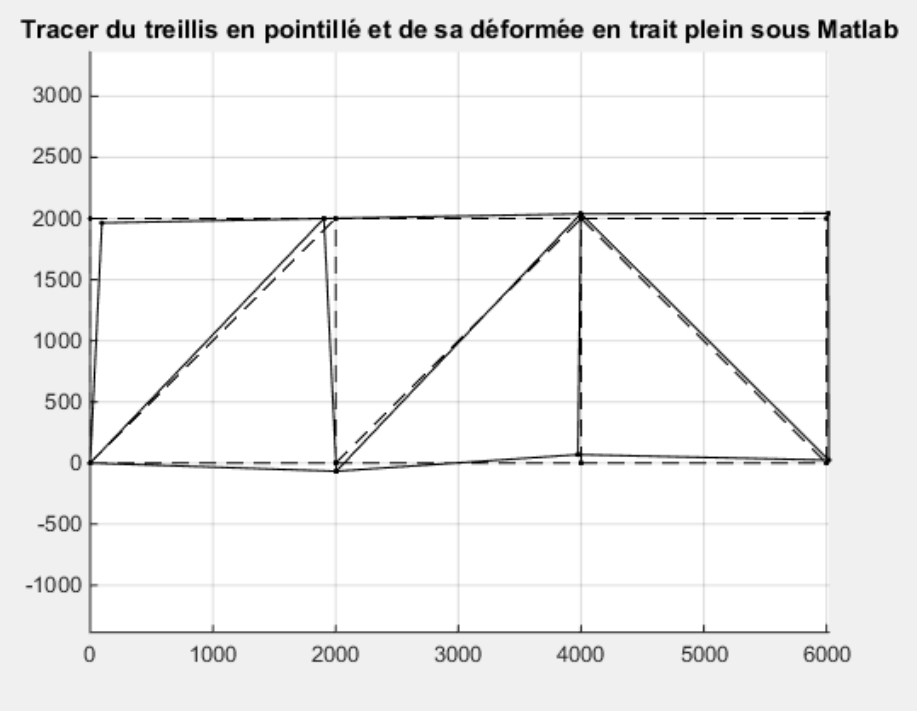

Figure 14. Mode 8.

$\lambda_{9}=2.71281519$

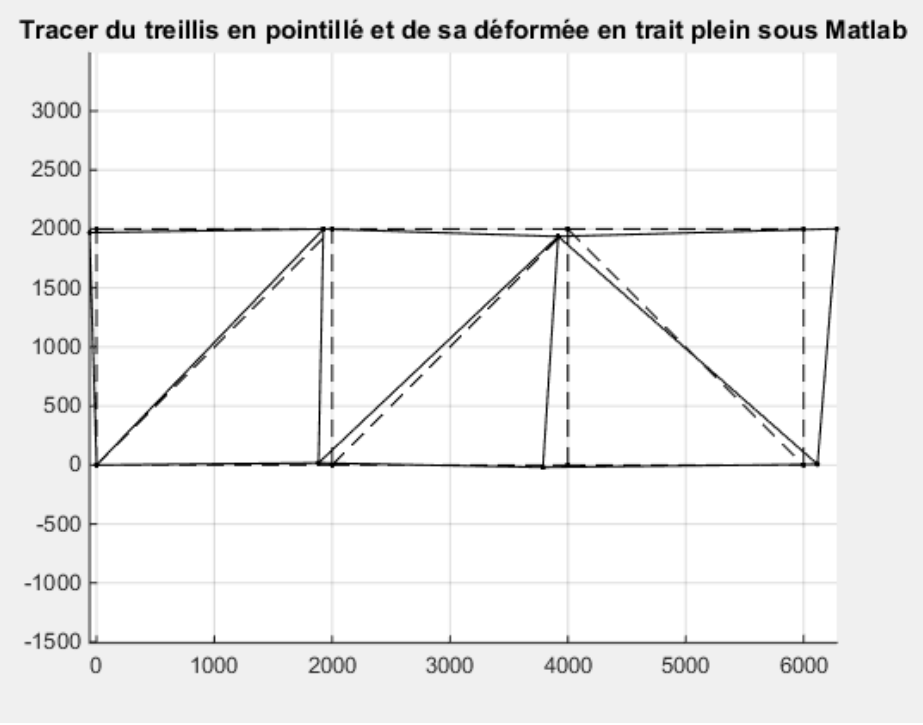

Figure 15. Mode 9.

$\lambda_{10}=1.2586962 \mathrm{D}$

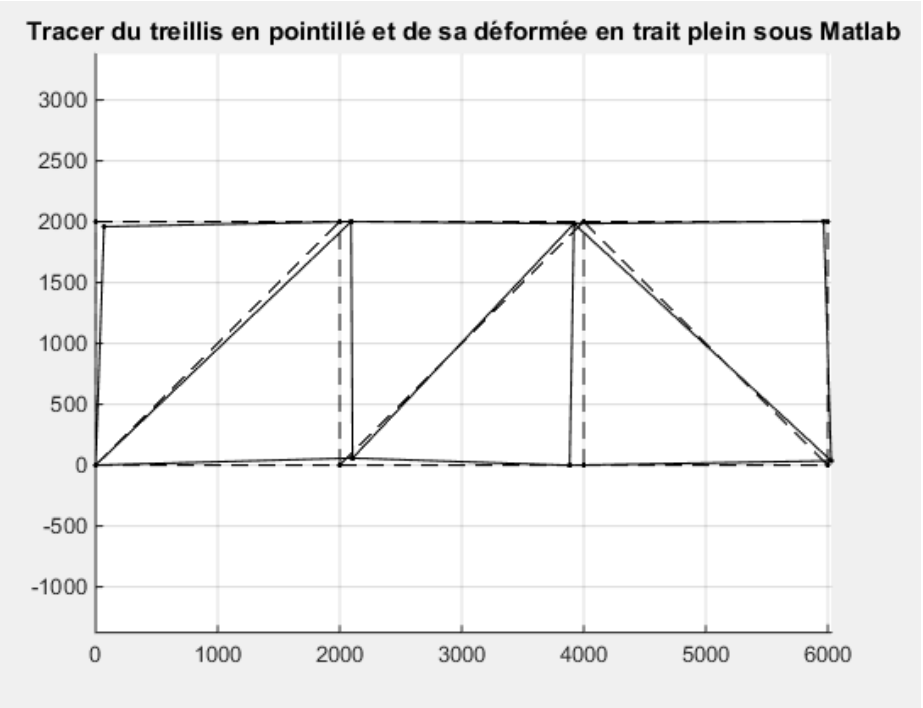

Figure 16. Mode 10.

$\lambda_{11}=0.854122378$ 


\section{Tracer du treillis en pointillé et de sa déformée en trait plein sous Matlab}

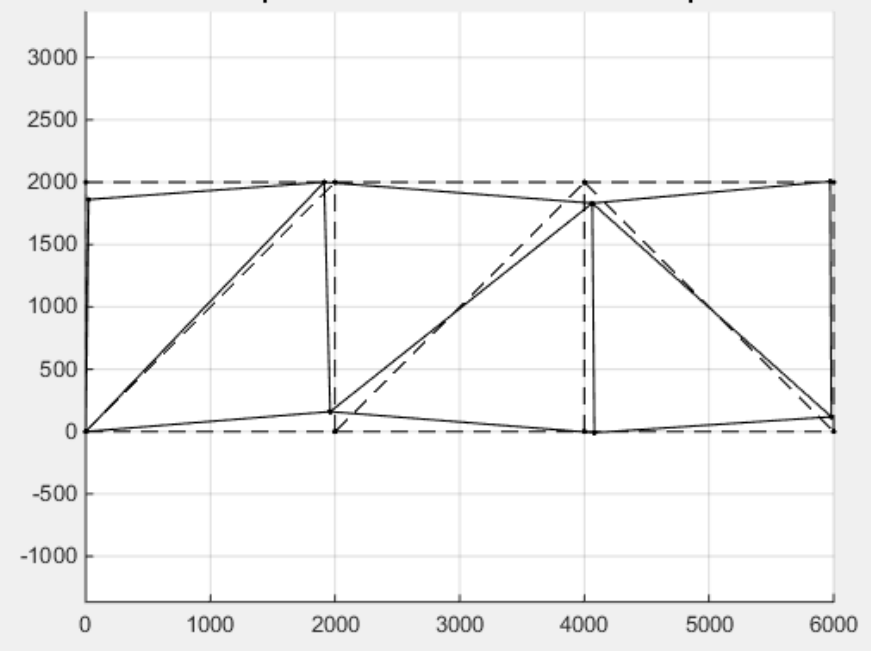

Figure 17. Mode 11.

$\lambda_{12}=0.306371047$

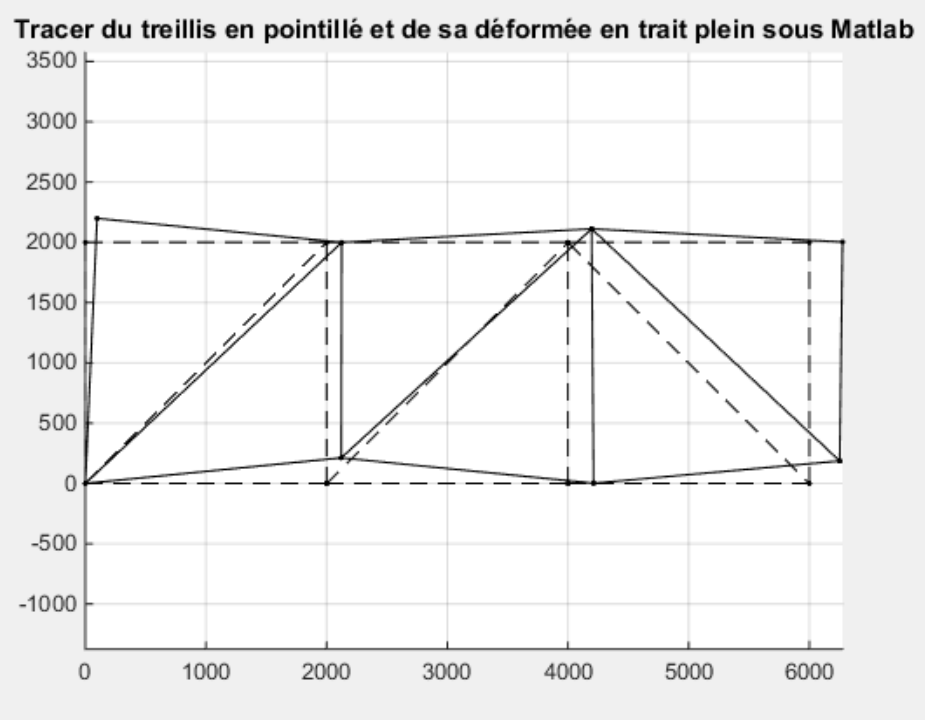

Figure 18. Mode 12.

$\lambda_{13}=0.1243095 \hbar$

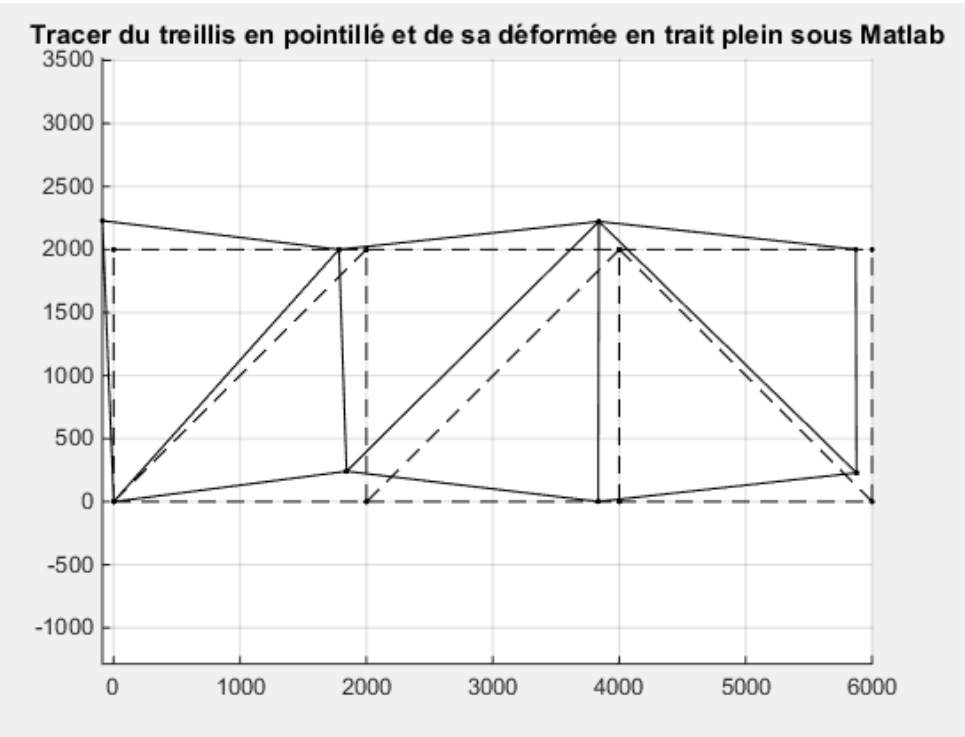

Figure 19. Mode 13 


\section{Conclusion}

Cet article montre l'importance de la méthode des éléments finis dans l'analyse modale des structures en treillis. Elle permet d'écrire les équations différentielles qui gouvernent les déplacements des nœuds du treillis. Pour effectuer l'analyse modale il est nécessaire de connaitre et d'écrire l'équation caractéristique du mouvement. L'équation différentielle du mouvement libre est permet d'obtenir l'équation caractéristique du système d'équation différentiel. L'outil Matlab a permis de résoudre cette équation caractéristique de treizièmes degrés. Pour chaque valeur propre, nous avons une pulsation propre et une déformation propre. Le logiciel Matlab a permis d'illustrer la déformation du treillis pour chaque mode de vibration. A cet effet, nous voyons de quelle façon la structure se déforme avant la rupture lorsqu'elle sollicitée par une fréquence égale à la fréquence modale.

\section{Bibliographie}

[GEN 11] GENEVAUX J.M., Dynamique des structures : méthodes approchées, cinématiques; analyse modale ; recalage de modèle, Ecole Nationale d'ingénieurs du Mans, 2011.

[BAT 90] BATOZ J.L ET DHATt G., Modélisation des structures par éléments finis, volume 1, Hemès, Paris, 1990.

[GER 95] GERADIN M. ET RIXEN D., théorie des vibrations : application à la dynamique des structures, Masson, 1995.

[GMU 97] GMUR T., Dynamique des structures : analyse modale numérique, Presses polytechniques romandes, 1997.

[DEC 97] DE COULON F., théorie et traitement des signaux, 1997.

[OTT 14] OTTO S.R ET DENIER J.P., An introduction to programming and numerical methods, school of mathematical sciences the university of Adelaide, south Australian.

[SUA 06] SUARD M., Caractérisation et optimisation de structure treillis fabriquées par EBM, Phd, Université de Grenoble, 2006.

[PRE 13] PREUMONT A., Twelve lecture on structural dynamics : active structures laboratory, Université Libre de Bruxelles, 2013.

[IMB 84] IMBER J.F., Analyses des structures par éléments finis, Cepadues Editions, 1984.

[DEH 02] DEHOMBREUX P., Finite element modale for mechanical engineering, Faculté polytechnique de Mons, 2002.

[SAN 69] SANDER G., application de la méthode des éléments finis à la flexion des plaques, thèse de doctorat, Université de Liège, 1969.

[HUC 76] HUCH A., Méthodes numérique en réponse dynamique avec amortissement structural, PhD, Université de Liège, 1976.

[DEL 84] Del PEDRo et PAHud P., Mécanique vibratoire, Presses polytechnique romandes, Lausanne, 1984.

[ZIE 77] ZIENKIEWIEZ C., The finite element method, Megraw hill, 1977.

[TOU 84] TOUZOT G. ET DhatT G., une presentation de la method des elements finis, Université de Campiège, Maloine, 1984. 